\title{
Constraints on Dark Energy Parameters from Correlations of CMB with LSS
}

\author{
Hong $\mathrm{Li}^{a, b}$ and Jun-Qing $\mathrm{Xia}^{c}$ \\ ${ }^{a}$ Institute of High Energy Physics, Chinese Academy of Science, P.O.Box 918-4, Beijing 100049, P.R.China \\ ${ }^{b}$ Theoretical Physics Center for Science Facilities (TPCSF), Chinese Academy of Science, P.R.China and \\ ${ }^{c}$ Scuola Internazionale Superiore di Studi Avanzati, Via Beirut 2-4, I-34014 Trieste, Italy
}

\begin{abstract}
In this paper, we combine the the latest observational data, including the WMAP five-year data (WMAP5), the baryon acoustic oscillations (BAO) and type Ia supernovae (SN) "union" compilation, and use the Markov Chain Monte Carlo method to determine the dark energy parameters. We pay particular attention to the Integrated Sache-Wolfe (ISW) data from the cross-correlations of cosmic microwave background (CMB) and large scale structure (LSS). In the $\Lambda$ CDM model, we find that the ISW data, as a complement to the WMAP data, could significantly improve the constraint of curvature $\Omega_{k}$. We also check the improvement of constraints from the new prior on the Hubble constant and find this new prior could improve the constraint of $\Omega_{k}$ by a factor of 2 . Finally, we study the dynamical evolving EoS of dark energy from the current observational data. Based on the dynamical dark energy model, parameterizing as $w(a)=w_{0}+w_{a}(1-a)$, we find that the $\Lambda \mathrm{CDM}$ model remains a good fit to the current data. When taking into account the ISW data, the error bars of $w_{0}$ and $w_{a}$ could be shrunk slightly. Current constraints on the dynamical dark energy model are not conclusive. The future precision measurements are needed.
\end{abstract}

\section{INTRODUCTION}

Unveiling the origin of the current accelerating expansion of our Universe is a big challenge for the modern cosmology either theoretically or observationally. The origin source which drives the expansion could be attributed to a mysterious budget, dark energy. Thus, the nature of dark energy is one of the biggest unsolved problems in modern physics and has been extensively investigated in recent years.

The measurements of CMB [1] 3], LSS surveys [4, 5] and SN [6, 7] have provided a lot of high quality data at present. These data have been widely used to constrain various cosmological models. However, one should keep in mind that the degeneracies of cosmological parameters generally exist in almost all cosmological observations, i.e., they are not sensitive to single parameters but to some specific combinations of them. These degeneracies could weaken constraints on the cosmological parameters. It is therefore highly necessary to combine different probes to break parameter degeneracies so as to achieve tight constraints. Furthermore, different observations are affected by different systematic errors, and it is thus helpful to reduce potential biases by combining different probes.

One of the useful complementary probe is the latetime ISW effect [8]. This ISW effect is produced by the CMB photons passing through the time-evolving gravitational potential well, when dark energy or curvature becomes important at later times. Therefore, the ISW effect provides a promising probe for studying the acceleration mechanism of our universe, especially for the dark energy and the curvature of Universe. Cross correlating CMB with tracers of LSS surveys for detecting the ISW effect 9] has been widely investigated in the literature [10 26].

In this paper, we will present the constraints on various cosmological models from the current observations, including the WMAP5 data, SN "union" compilation, and recently released BAO data from SDSS DR7, as well as the ISW data. The structured of the paper is as following: in Section II we describe the method and the data sets; in Section III we present our numerical results and discussion; finally we give a summary and outlook in Section VI.

\section{METHOD AND DATA}

For the parametrizations of dark energy models, we adopt $\Lambda$ CDM model and dynamical dark energy model with CPL parametrization [27] as following:

$$
w_{\mathrm{DE}}(a)=w_{0}+w_{a}(1-a)
$$

where $a=1 /(1+z)$ is the scale factor and $w_{a}$ characterizes the "running" of the equation-of-state $(\mathrm{EoS})$ of dark energy. In the $\Lambda \mathrm{CDM}$ model, $w_{0}=-1$ and $w_{a}=0$.

For dark energy models whose EoS is not equal to -1 during the evolution of Universe, the perturbation of dark energy will inevitably exist. The perturbation of dark energy has no effect on the geometric constraints. However, when including the CMB and LSS data, the perturbation of dark energy should be fully considered, because the late time ISW effect will be different significantly, and will take an important signature on large angular scales of CMB and the matter power spectrum of LSS [28]. For quintessence-like or phantom-like models, in which $w$ does not cross the cosmological constant boundary, the perturbation of dark energy is well defined. However, when $w$ crosses -1 , one is encountered with the divergence problem for perturbations of dark energy at $w=-1$. In order to solve this problem in the global analysis, we introduce a small positive constant $\epsilon$ to divide the full range of the allowed value of the EoS $w$ into three parts: 1) $w>-1+\epsilon$; 2) $-1-\epsilon \leq w \leq-1+\epsilon$; and 3) $w<-1-\epsilon$. 

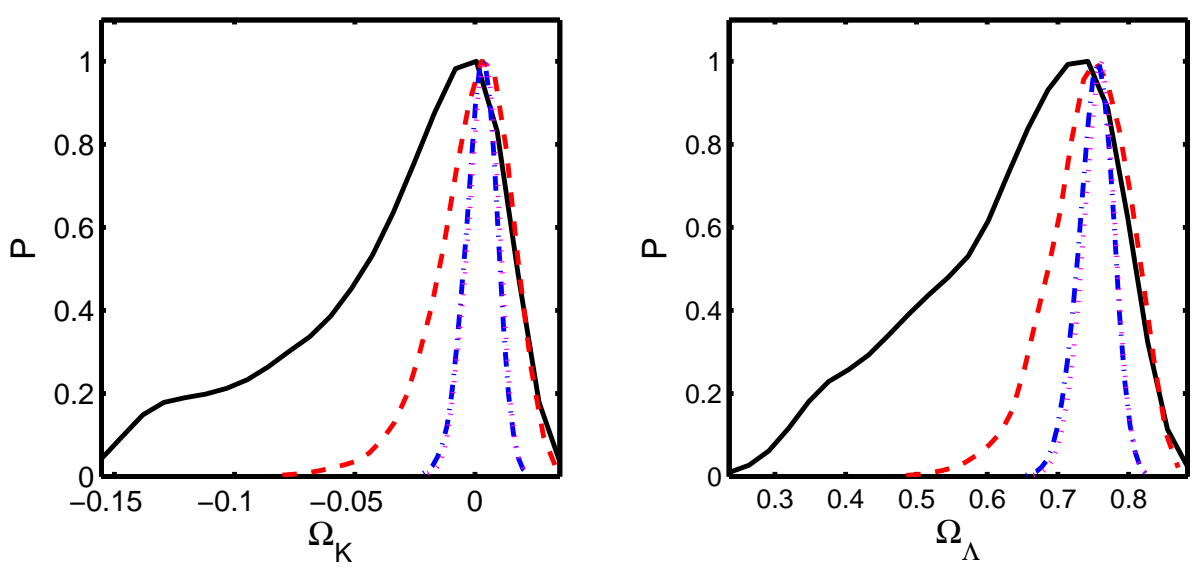

FIG. 1: One dimensional distributions of $\Omega_{k}$ and $\Omega_{\Lambda}$ from different data combinations: WMAP5 (balck solid lines), WMAP5+ISW (red dashed lines), WMAP5+HST (blue dash-dotted lines), and WMAP5+HST+ISW (purple dotted lines).

Working in the conformal Newtonian gauge, the perturbations of $\mathrm{DE}$ can be described by

$$
\begin{aligned}
\dot{\delta} & =-(1+w)(\theta-3 \dot{\Phi})-3 \mathcal{H}\left(c_{s}^{2}-w\right) \delta, \\
\dot{\theta} & =-\mathcal{H}(1-3 w) \theta-\frac{\dot{w}}{1+w} \theta+k^{2}\left(\frac{c_{s}^{2} \delta}{1+w}+\Psi\right) .
\end{aligned}
$$

Neglecting the entropy perturbation, for the regions 1) and 3), the EOS does not across -1 and the perturbation is well defined by solving Eqs.(223). For the case 2), the perturbation of energy density $\delta$ and divergence of velocity, $\theta$, and the derivatives of $\delta$ and $\theta$ are finite and continuous for the realistic dark energy models. However for the perturbations of the parameterizations, there is clearly a divergence. In our analysis for such a regime, we match the perturbations in region 2) to the regions 1) and 3) at the boundary and set $\dot{\delta}=0$ and $\dot{\theta}=0$. In our numerical calculations we limit the range to be $|\Delta w=\epsilon|<10^{-4}$ and find our method to be a very good approximation to the multi-field dark energy model. More detailed treatments can be found in Ref.[28].

The publicly available Markov Chain Monte Carlo (MCMC) package CosmoMC ${ }^{1}$ 29] is employed in our global fitting, and modifications have been made to include dark energy perturbations, and to suit the dark energy models which we study [28, 30]. Furthermore, we assume purely adiabatic initial conditions in our calculations.

Our most general theory parameter space vector is:

$$
\mathbf{P} \equiv\left(\omega_{b}, \omega_{c}, \Theta_{s}, \tau, w_{0}, w_{a}, \Omega_{k}, n_{s}, A_{s}, c_{s}^{2}\right)
$$

where $\omega_{b} \equiv \Omega_{b} h^{2}$ and $\omega_{c} \equiv \Omega_{c} h^{2}$, in which $\Omega_{b}$ and $\Omega_{c}$ are the physical baryon and cold dark matter densities relative to the critical density, $\Omega_{k}$ is the spatial curvature and satisfies $\Omega_{k}+\Omega_{m}+\Omega_{\Lambda}=1, \Theta_{s}$ is the ratio

\footnotetext{
${ }^{1}$ Available at: http://cosmologist.info/cosmomc/
}

(multiplied by 100) of the sound horizon to the angular diameter distance at decoupling, $\tau$ is the optical depth to re-ionization, $w_{0}$ and $w_{a}$ are the parameters of dark energy EoS given by Eq.(11), $A_{s}$ and $n_{s}$ characterize the power spectrum of primordial scalar perturbations, $c_{s}^{2}$ is the sound speed of dark energy. For the pivot scale we set $k_{s 0}=0.05 \mathrm{Mpc}^{-1}$.

In the computation of CMB we have included the WMAP5 temperature and polarization power spectra with the routine for computing the likelihood supplied by the WMAP team ${ }^{2}$. We also combine the distance measurements from BAO and SNIa. For the BAO information, we use the newly released gaussian priors on the distance ratios, $r_{s} / D_{v}(z)=0.1905 \pm 0.0061$ at $z=0.2$ and $r_{s} / D_{v}(z)=0.1097 \pm 0.0036$ at $z=0.35$, with a correlation coefficient of 0.337 , which were measured from the power spectrum for the distribution of the spectroscopic Sloan Digital Sky Survey (SDSS) Data Release 7 (DR7) galaxy sample and the 2-degree Field Galaxy Redshift Survey (2dFGRS) data 33]. For the calculation of the likelihood from supernova, we use are the "Union" compilation (307 sample) 6] and have marginalized over the nuisance parameter [31].

For the ISW data, we have included the package for calculating the ISW likelihood function provided by Ref.[23], which contain a $3.7 \sigma$ detection of ISW by cross correlating WMAP data with the LSS data sets of 2Micron All Sky Survey sample, SDSS photometric Luminous Red Galaxies, SDSS photometric quasars, and NRAO VLA Sky Survey radio sources. In our calculations, we have taken the total likelihood to be the products of the separate likelihoods $\mathcal{L}$ of $\mathrm{CMB}, \mathrm{BAO}$, SN and ISW. Furthermore, we make use of the newly released HST prior on the Hubble constant, which is

\footnotetext{
2 Available at the LAMBDA website: http://lambda.gsfc.nasa.gov/
} 
the measurement of the Hubble parameter by the Near Infrared Camera and Multi-Object Spectrometer (NICMOS) Camera 2 of the Hubble Space Telescope (HST) and give $H_{0} \equiv 100 \mathrm{~h} \mathrm{~km} \mathrm{~s}^{-1} \mathrm{Mpc}^{-1}$ by a Gaussian likelihood function centered around $H_{0}=74.2$ and with a standard deviation $\sigma=3.8$ [32].

\section{NUMERICAL RESULTS}

In this section we present our global fitting results of the cosmological parameters determined from the latest observational data.

In Table I we list the constraints on the dark energy density $\Omega_{\Lambda}$ and the curvature $\Omega_{k}$ in the $\Lambda \mathrm{CDM}$ model from different data combinations. Due to the well-known degeneracy between $\Omega_{\Lambda}$ and $\Omega_{k}$, we obtain a weak constraint on the curvature $\Omega_{k}$ from WMAP5 data only, $\Omega_{k}=-0.036 \pm 0.043$ ( $68 \%$ C.L.), as shown in Fig [1 Our universe is very close to flatness, which is consistent with the prediction of inflation paradigm. However, this degeneracy could be broken by adding other different cosmological data, such as the large scale structure and supernovae data. When we add the ISW data into the analysis, we can find that the combined constraint from WMAP $5+$ ISW is significantly improved over using WMAP5 alone, namely the $68 \%$ interval is $\Omega_{k}=-0.002 \pm 0.016$. The constraints of $\Omega_{k}$ and $\Omega_{\Lambda}$ improve by a factor of 2.6 and 2.2 , respectively. The ISW data give the remarkable complementary effect on WMAP5 data in constraining cosmological parameters and break the degeneracy between $\Omega_{k}$ and $\Omega_{\Lambda}$.

In Fig 1 we also plot the one dimensional posterior distribution of $\Omega_{k}$ and $\Omega_{\Lambda}$ from WMAP $5+$ HST prior. Here, we consider the new released HST prior, $h=0.742 \pm 0.038$ $(1 \sigma)$. We find that this new HST prior could play an important role in constraining cosmological parameter and give much tighter constraints on cosmological parameters, $\Omega_{k}=0.002 \pm 0.007(1 \sigma)$. The error bar has been shrunk by a factor of 6.6 when compared to the constraint from WMAP 5 alone. The $95 \%$ intervals of $\Omega_{k}$ and $\Omega_{\Lambda}$ are $-0.012<\Omega_{k}<0.014$ and $0.70<\Omega_{\Lambda}<0.80$. We also do the calculations using the old HST prior, $h=0.72 \pm 0.08$ [34]. Using WMAP $5+$ HST old prior, we obtain that the $2 \sigma$ constraints on $\Omega_{k}$ and $\Omega_{\Lambda}$ are $-0.045<\Omega_{k}<0.015$ and $0.59<\Omega_{\Lambda}<0.80$, respectively. The constraints significantly improve by a factor of 2 . Furthermore, when combining WMAP5, ISW and HST together, the constraint on $\Omega_{k}$ will improve further: $\Omega_{k}=0.003 \pm 0.006$ at $68 \%$ confidence level.

In Fig 2 we present the two dimensional contours of $\Omega_{k}$ and $\Omega_{\Lambda}$ from different data combinations in the $\Lambda$ CDM model. We find that $\Omega_{k}$ and $\Omega_{\Lambda}$ are highly correlated with each other. The ISW data and the new HST prior are very helpful in breaking such degeneracy.

We now present the constraints on dark energy parameters. In Ref. 23], they considered the constraints on dark energy model with constant EoS, and found that

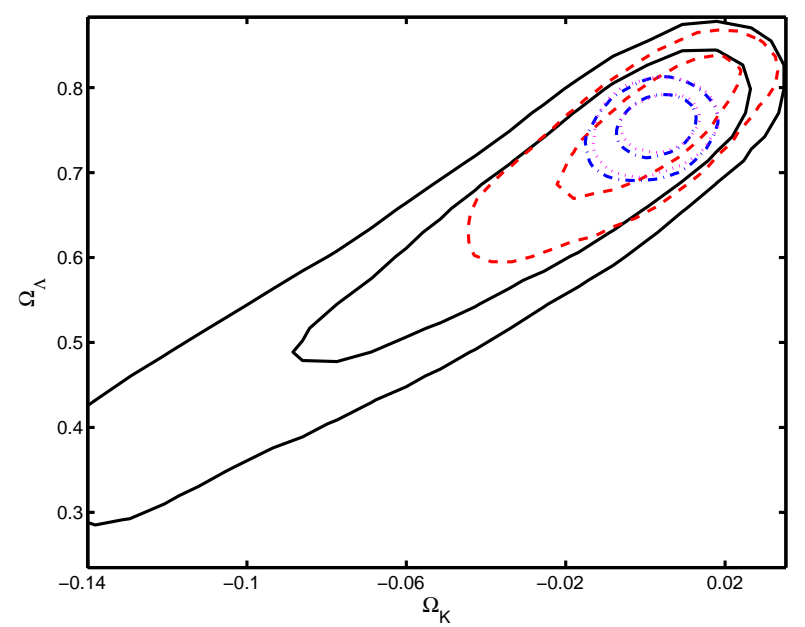

FIG. 2: Two dimensional contours of $\Omega_{k}$ and $\Omega_{\Lambda}$ from different data combinations within the $\Lambda$ CDM model: WMAP5 (balck solid lines), WMAP5+ISW (red dashed lines), WMAP5+HST (blue dash-dotted lines), and WMAP5+HST+ISW (purple dotted lines).

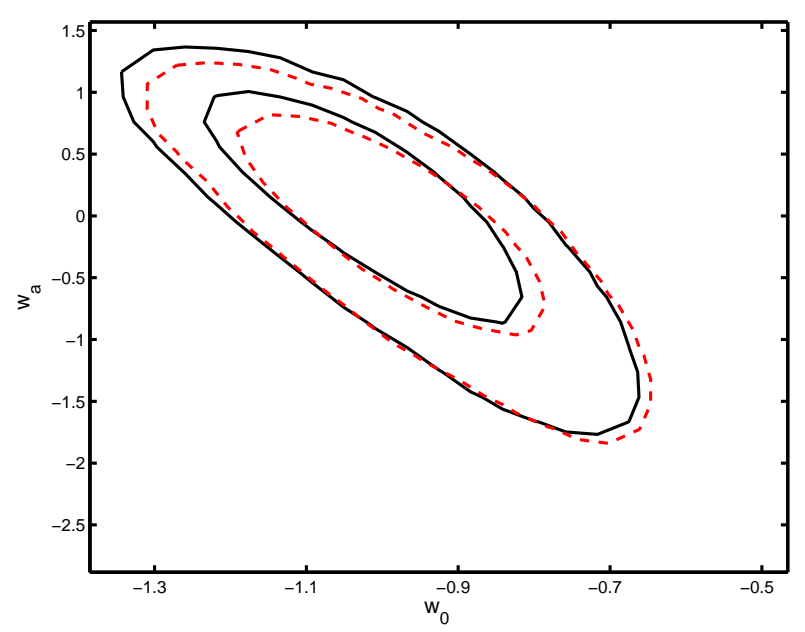

FIG. 3: Two dimension contours of the EoS parameters of dark energy $w_{0}$ and $w_{a}$. The black solid lines are given by WMAP $5+\mathrm{SN}+\mathrm{BAO}$ and the red dashed lines are given by WMAP5+SN+BAO+ISW, respectively.

the ISW data could modestly improve constraint of dark energy EoS. As we know, the variation of dark energy EoS could also affect the evolution of gravitational potential, and the ISW effect consequently. It would be worth checking the capabilities of ISW data on the constraint of time-varying dark energy EoS. In Fig 3 we plot the two dimensional contours of $w_{0}$ and $w_{a}$ from different data combinations. The black solid lines are given by fitting with the combination of WMAP 5 temperature and polarization power spectra, supernovae data, as well as the newly released BAO data. Due to the limits of the precisions of observational data, the variance of $w_{0}$ and 
TABLE I. Mean $1 \sigma$ constraints on $\Omega_{k}$ and $\Omega_{\Lambda}$ from different data combinations in the $\Lambda$ CDM model.

\begin{tabular}{c|c|c|c|c}
\hline \hline & WMAP only & WMAP+ISW & WMAP+HST & WMAP+ISW+HST \\
\hline $100 \times \Omega_{k}$ & $-3.57 \pm 4.28$ & $-0.182 \pm 1.61$ & $0.226 \pm 0.65$ & $0.304 \pm 0.630$ \\
\hline$\Omega_{\Lambda}$ & $0.635 \pm 0.130$ & $0.742 \pm 0.0566$ & $0.752 \pm 0.0243$ & $0.756 \pm 0.0228$ \\
\hline \hline
\end{tabular}

TABLE II. Mean $1 \sigma$ constraints on $w_{0}, w_{a}$ and $\Omega_{\Lambda}$ from different data combinations in the dynamical dark energy model.

\begin{tabular}{c|c|c}
\hline \hline & WMAP+SN+BAO & WMAP+SN+BAO+ISW \\
\hline$w_{0}$ & $-1.002 \pm 0.136$ & $-0.970 \pm 0.130$ \\
\hline$w_{a}$ & $0.0096 \pm 0.64$ & $-0.134 \pm 0.612$ \\
\hline$\Omega_{\Lambda}$ & $0.732 \pm 0.0149$ & $0.734 \pm 0.0144$ \\
\hline \hline
\end{tabular}

$w_{a}$ are still large, namely the $95 \%$ constraints on $w_{0}$ and $w_{a}$ are $-1.25<w_{0}<-0.72$ and $-1.47<w_{a}<1.00$ respectively, which are listed in Table II. This result implies that the dynamical dark energy models are not excluded and the current data cannot distinguish different dark energy models decisively. The $\Lambda$ CDM model, however, is still a good fit right now.

We also include the ISW data into the calculations. From the red dashed lines in Fig 3, we can find that the constraints on the dark energy EoS parameters slightly improve from WMAP $5+\mathrm{SN}+\mathrm{BAO}+\mathrm{ISW}$ combination. The $95 \%$ intervals are $-1.21<w_{0}<-0.71$ and $-1.52<$ $w_{a}<0.86$. The ISW data help tighten the lower limit on $w_{0}$ and the upper limit on $w_{a}$, but not significantly. These is due to the constraining power of SNIa and BAO, while the current ISW data are still not constraining enough. However, the ISW data could be a useful probe for constraining other dark energy models, such as the early dark energy model [25]. And they also found that the future ISW data can improve the constraints on the most important cosmological parameters by a factor $\sim 1.5$.

Again, we also compare the constraints on dark energy parameters between from the new HST prior and from the old one. When we combine WMAP5, SN, BAO, as well as the old HST prior, we obtain that the $2 \sigma$ constraints on $w_{0}$ and $w_{a}$ are $-1.23<w_{0}<-0.7$ and $-1.49<w_{a}<0.94$, which is slightly weaker than those from the new HST prior.

If dark energy is not the cosmological constant, we should consider the dark energy perturbations in our calculations. In the framework of the linear perturbations theory, besides the EoS of dark energy, the dark energy perturbations can also be characterized by the sound speed, $c_{s}^{2} \equiv \delta p_{\Lambda} / \delta \rho_{\Lambda}$. The sound speed of dark energy affects the evolution of perturbations, and leaves the signatures on the CMB power spectrum [35, 38, 39]. Thus, in the literature constraining on the sound speed $c_{s}^{2}$ from different observational data has been widely investigated (e.g. Refs. [36, 37, 39]).

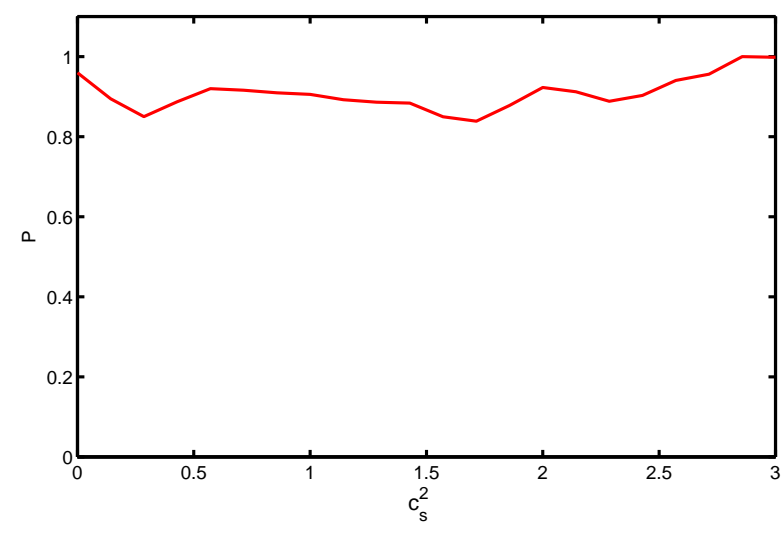

FIG. 4: One dimensional constraints on the sound speed $c_{s}^{2}$ from WMAP $5+\mathrm{SN}+\mathrm{BAO}+\mathrm{ISW}$ combination in the dynamical dark energy model.

Here, we also constrain the dark energy sound speed from current data in the dynamical dark energy model. In Fig 4, we show the one dimensional constraint on the dark energy sound speed $c_{s}^{2}$ from the combination of WMAP5 temperature and polarization power spectra, SNIa and BAO, as well as the ISW data. We can find that the constraint on the dark energy sound speed $c_{s}^{2}$ are still very weak. The current observational data are still not accurate enough.

\section{SUMMARY}

In this paper, we study the constraints on cosmological parameters from the recently released $\mathrm{CMB}, \mathrm{BAO}$ and SNIa data. Here, we pay particular attention to the current ISW data which is the cross correlations of CMB with LSS surveys. In the $\Lambda$ CDM model, the ISW data and the new HST prior are very helpful to break the degeneracy between $\Omega_{k}$ and $\Omega_{\Lambda}$. The constraints on $\Omega_{k}$ and $\Omega_{\Lambda}$ significantly improve, when compared to the constraints from WMAP5 alone.

More importantly, we consider the constraints on the dark energy parameters in the CPL dark energy model. Here, we fully include the perturbations of dark energy. This result implies that the dynamical dark energy models are not excluded, the $\Lambda$ CDM model, however, is still a good fit right now. We find that the ISW data could give slight improvement of the constraints on CPL dark energy model. But this does not mean that the ISW data can not constrain the dynamical dark energy models effi- 
ciently. Actually, it should be useful for constraining the dynamical dark energy models whose $\operatorname{EoS} w(z)$ deviate from the cosmological constant boundary obviously, for example, the early dark energy models [25], or the dark energy model with its EoS $w(z)$ transits sharply during its evolution. Besides the dark energy models, the ISW data could be also helpful for testing the modified gravity theories 40 43], massive neutrinos [44], the primordial non-gaussianity [45, 46], and so on.

Furthermore, we compare the constraints between from the new HST prior and from the old one. One can see that the new HST prior gives the tighter constraints on the cosmological parameters. Finally, we check the capability of current observational data to constrain the dark energy sound speed $c_{s}^{2}$. We find that the sound speed is weakly constrained by current observations, and thus futuristic precision measurements of the $\mathrm{CMB}$ on a very large angular scale (low multipoles) are necessary.

\section{Acknowledgements}

We acknowledge the use of the Legacy Archive for Microwave Background Data Analysis (LAMBDA). Support for LAMBDA is provided by the NASA Office of Space Science. Our numerical analysis was performed on the MagicCube of Shanghai Supercomputer Center (SSC). We thank Yi-Fu Cai for helpful discussions. This work is supported in part by the National Natural Science Foundation of China under Grant No. 10803001, 973 Program under Grant No.2010CB833000 and the Youth Foundation of the institute of high energy physics under Grant Nos. H95461N.
[1] E. Komatsu et al. [WMAP Collaboration], Astrophys. J. Suppl. 180, 330 (2009).

[2] J. Dunkley et al. [WMAP Collaboration], Astrophys. J. Suppl. 180, 306 (2009).

[3] G. Hinshaw et al. Astrophys. J. Suppl. 180, 225 (2009).

[4] M. Tegmark, et al., Astrophys. J. 606, 702 (2004); M. Tegmark, et al., Phys. Rev. D 74, 123507 (2006).

[5] S. Cole, et al., Mon. Not. R. Astron. Soc. 362, 505 (2005).

[6] M. Kowalski et al. Astrophys. J. 686, 749 (2008).

[7] M. Hicken et al., Astrophys. J. 700, 1097 (2009).

[8] R. K. Sachs and A. M. Wolfe, Astrophys. J. 147, 73 (1967).

[9] R. G. Crittenden and N. Turok, Phys. Rev. Lett. 76, 575 (1996) arXiv:astro-ph/9510072.

[10] S. P. Boughn, R. G. Crittenden and N. G. Turok, New Astron. 3, 275 (1998) arXiv:astro-ph/9704043.

[11] M. Zaldarriaga and U. Seljak, Phys. Rev. D 59, 123507 (1999) arXiv:astro-ph/9810257.

[12] W. Hu and T. Okamoto, Astrophys. J. 574, 566 (2002) arXiv:astro-ph/0111606.

[13] Y. S. Song, A. Cooray, L. Knox and M. Zaldarriaga, Astrophys. J. 590, 664 (2003) arXiv:astro-ph/0209001.

[14] W. Hu, Phys. Rev. D 65, $023003 \quad$ (2002) arXiv:astro-ph/0108090.

[15] W. Hu, Astrophys. J. 557, L79 (2001) arXiv:astro-ph/0105424.

[16] N. Afshordi, Y. S. Loh and M. A. Strauss, Phys. Rev. D 69, 083524 (2004) arXiv:astro-ph/0308260.

[17] E. Gaztanaga, M. Manera and T. Multamaki, Mon. Not. Roy. Astron. Soc. 365, 171 (2006) arXiv:astro-ph/0407022.

[18] P. Vielva, E. Martinez-Gonzalez and M. Tucci, arXiv:astro-ph/0408252

[19] D. Pietrobon, A. Balbi and D. Marinucci, Phys. Rev. D 74, 043524 (2006) arXiv:astro-ph/0606475.

[20] J. D. McEwen, P. Vielva, M. P. Hobson, E. MartinezGonzalez and A. N. Lasenby, Mon. Not. Roy. Astron. Soc. 373, 1211 (2007) arXiv:astro-ph/0602398.

[21] T. Giannantonio et al., Phys. Rev. D 74, 063520 (2006) arXiv:astro-ph/0607572.

[22] A. Rassat, K. Land, O. Lahav and F. B. Ab- dalla, Mon. Not. Roy. Astron. Soc. 377, 1085 (2007) arXiv:astro-ph/0610911.

[23] S. Ho, C. Hirata, N. Padmanabhan, U. Seljak and N. Bahcall, Phys. Rev. D 78, 043519 (2008) arXiv:0801.0642 [astro-ph]].

[24] B. R. Granett, M. C. Neyrinck and I. Szapudi, arXiv:0805.3695 [astro-ph].

[25] J. Q. Xia, M. Viel, C. Baccigalupi and S. Matarrese, arXiv:0907.4753 [astro-ph.CO].

[26] L. Pogosian, P. S. Corasaniti, C. Stephan-Otto, R. Crittenden and R. Nichol, Phys. Rev. D 72, 103519 (2005) arXiv:astro-ph/0506396.

[27] M. Chevallier and D. Polarski, Int. J. Mod. Phys. D 10, 213 (2001); E. V. Linder, Phys. Rev. Lett. 90, 091301 (2003).

[28] G. B. Zhao, J. Q. Xia, M. Li, B. Feng and X. Zhang, Phys. Rev. D 72, 123515 (2005) arXiv:astro-ph/0507482.

[29] A. Lewis and S. Bridle, Phys. Rev. D 66, 103511 (2002).

[30] J. Q. Xia, G. B. Zhao, B. Feng, H. Li and X. Zhang, Phys. Rev. D 73, 063521 (2006) arXiv:astro-ph/0511625.

[31] E. Di Pietro and J. F. Claeskens, Mon. Not. Roy. Astron. Soc. 341, 1299 (2003).

[32] A. G. Riess et al., Astrophys. J. 699, 539 (2009) arXiv:0905.0695 [astro-ph.CO]].

[33] W. J. Percival et al., arXiv:0907.1660 [astro-ph.CO].

[34] W. L. Freedman, et al., Astrophys. J. 553, 47 (2001).

[35] J. Weller and A. M. Lewis, Mon. Not. Roy. Astron. Soc. 346, 987 (2003) arXiv:astro-ph/0307104.

[36] S. DeDeo, R. R. Caldwell and P. J. Steinhardt, Phys. Rev. D 67, 103509 (2003) [Erratum-ibid. D 69, 129902 (2004)] arXiv:astro-ph/0301284.

[37] J. K. Erickson, R. R. Caldwell, P. J. Steinhardt, C. Armendariz-Picon and V. F. Mukhanov, Phys. Rev. Lett. 88, 121301 (2002) arXiv:astro-ph/0112438.

[38] W. Hu and R. Scranton, Phys. Rev. D 70, 123002 (2004) arXiv:astro-ph/0408456.

[39] J. Q. Xia, Y. F. Cai, T. T. Qiu, G. B. Zhao and X. Zhang, Int. J. Mod. Phys. D 17, 1229 (2008) arXiv:astro-ph/0703202.

[40] B. Jain and P. Zhang, Phys. Rev. D 78, 063503 (2008) arXiv:0709.2375 [astro-ph]]. 
[41] N. Afshordi, Phys. Rev. D 70, $083536 \quad$ (2004) arXiv:astro-ph/0401166.

[42] P. Zhang, Phys. Rev. D 73, 123504 (2006) arXiv:astro-ph/0511218.

[43] F. Schmidt, M. Liguori and S. Dodelson, Phys. Rev. D 76, 083518 (2007) arXiv:0706.1775 [astro-ph]].
[44] J. Lesgourgues, W. Valkenburg and E. Gaztanaga, Phys. Rev. D 77, 063505 (2008) arXiv:0710.5525 [astro-ph]].

[45] A. Slosar et al., JCAP 0808, 031 (2008).

[46] J. Q. Xia et al., arXiv:1003.3451. 